\title{
THE DUAL-BASELINE INTERFEROMETRIC PROCESSING CHAIN FOR THE TANDEM-X MISSION
}

\author{
Marie Lachaise, Ulrich Balss, Thomas Fritz, Helko Breit \\ Remote Sensing Technology Institute IMF \\ German Aerospace Center DLR \\ D-82230 Wessling
}

\begin{abstract}
During the second operational year of the TanDEM-X mission, a second coverage of the whole land mass is acquired in order to produce a high accurate and high resolution DEM from a combination of both data sets. This paper presents the dual-baseline interferometric processing chain. Its main steps consist of coregistering the different interferograms (having different baselines), of unwrapping the phases and of comparing them to eliminate the possible unwrapping errors.
\end{abstract}

Index Terms - Dual-baseline interferometric chain, InSAR, TanDEM-X Mission, phase unwrapping error correction, clapboard pattern

\section{INTRODUCTION}

The German TanDEM-X mission, started on June $21^{\text {st }}$, 2010, is based on two similar satellites flying in close formation and establishing the first bistatic single pass interferometer in space. The primary objective of the mission is the generation of a consistent global digital elevation model (DEM) with an unprecedented accuracy [1]. To achieve this accuracy, the whole land mass will be mapped at least twice, the first coverage has been acquired in 2011 and the second has already started. They have different heights of ambiguity to allow dual-/multi-baseline phase unwrapping. For the sake of a homogeneous data quality, the second acquisition is shifted by half the swath width with respect to the first coverage which increases the difficulty of the dual-baseline interferometric chain. Finally, difficult terrain will be covered some more times with different geometry (i.e. different look direction and/or incidence angle). Systematic processing of SAR raw data to so-called raw DEMs is performed by one single processing system, the Integrated TanDEM-X Processor (ITP, [2]) which includes the processing of the dual-/multi-baseline data. This paper presents an overview of the dual-baseline InSAR chain of ITP i.e. the part of the interferometric processing chain specific for the second mission year. It does not further explain the phase unwrapping correction algorithm (see [3] for more details) but is dedicated to the different processing steps.

\section{DUAL-BASELINE CHAIN OVERVIEW}

\subsection{Challenges statement}

The dual-baseline interferometric chain is used during the second TanDEM-X mission year.

In the following, the second year acquisition and its relative data are called master data and the first year acquisition is referred as slave data because they are coregistered to the master data.

Second year data have the same acquisition geometry (i.e. incidence angle) but different baseline respectively height of ambiguity. Typical values for the heights of ambiguity $\left(h_{2 \pi}\right)$ are 45 to $50 \mathrm{~m} /$ cycle for the first year and 30 to $35 \mathrm{~m} /$ cycle for the second year giving a height of ambiguity ratio $\alpha$ of

$$
\alpha=\frac{h_{2 \pi}^{m}}{h_{2 \pi}^{S}} \approx 0.7
$$

The second year acquisition is shifted by half the swath width in order to get a homogeneous quality forming a socalled clapboard pattern (Figure 1). It results that every scene acquired during the second mission year is covered by at least two half scenes acquired during the first mission year. This acquisition plan implies several difficulties regarding dual-baseline phase unwrapping. First, the time
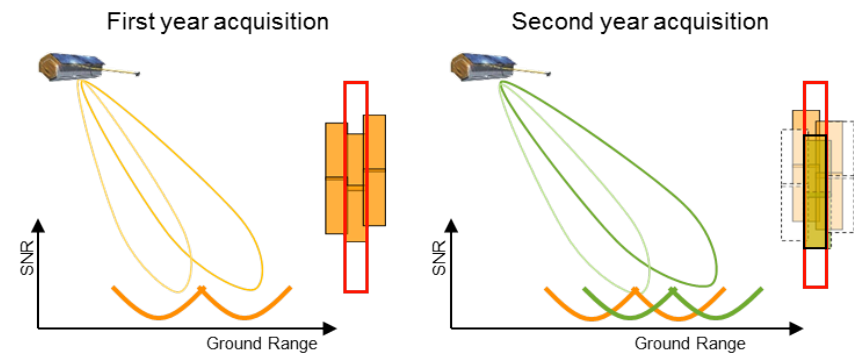

Figure 1: clapboard pattern and the scene overlap scheme: the second year is shifted by half the swath width to get a homogeneous quality. 
span between both data being about one year, it is not possible to use conventional multi-baseline phase unwrapping methods [4] due to the high temporal decorrelation. Second, the clapboard pattern brings that overlapping data do not have the same SNR or coherence since the best quality of one scene is covered by the part of worst SNR or coherence of the two other scenes.

\subsection{Key steps of the chain}

The dual-baseline chain is fully integrated in the ITP and is activated as soon as some CoSSCs (Coregistered Singlelook Slant-range Complex, i.e. pairs of coregistered images) processed during the first mission year are provided to support the interferometric processing of the second year master - data. The chain can handle as many supporting CoSSCs - slaves - as available as long as they overlap and have a sufficiently different baseline and the same acquisition geometry. The whole dual-baseline processing is done for every CoSSC pair separately. It consists of (Figure 2 and Figure 3):

- its coregistration on the master interferogram $\left(I_{m}\right)$,

- its cropping to the common part with $I_{m \text {, }}$

- forming the slave interferogram $I_{s}$ and unwrapping it,

- checking phase unwrapping consistency of both master and slave unwrapped phases (in heights),

- if phase unwrapping is inconsistent, calculation of the differential interferogram $\left(I_{d}\right)$ and possibly its correction with the stereo-radargrammetric phase followed by the correction of the master unwrapped phase.

A final overall step is the mosaicking of the different corrected master unwrapped phases (one corrected phase for every overlap with a different slave interferogram).

\section{THE DIFFERENT PROCESSING STEPS}

\subsection{Preparation of the supporting CoSSCs}

Since we have Coregistered SSCs and a master interferogram (and therefore already coregistered), we only need to coregister both master SSCs and then apply the resampling matrix to both channels of the CoSSC. However, since there is a time span of about one year between both acquisitions, the usual ITP coregistration algorithm based on coherent cross-correlation i.e. on signal [5] cannot be used because of the high temporal decorrelation.

Here, it is necessary to recall that the data are bistatic. Furthermore, the usual ITP coregistration uses geometrical shift estimates as a priori for the final precise signal coregistration. When we coregister the first-year data to the second-year data, we form a repeat pass monostatic pair. Since all the data have the same acquisition geometry but different baselines, it is possible to use the signal based coregistration shifts from the bistatic second-year acquisition and to rescale them to some second-first year

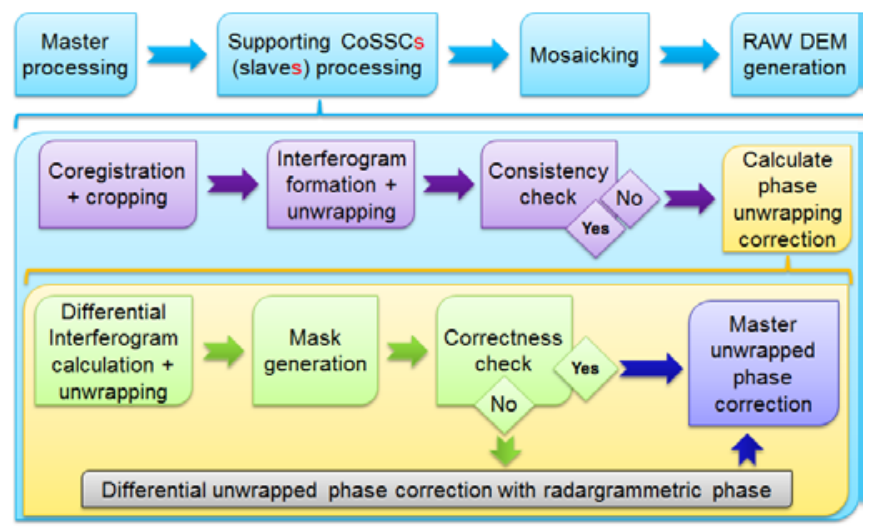

Figure 2: Overview diagram of the whole dual-baseline interferometric chain inside the ITP

monostatic shifts using geometry. Indeed, these coregistration shifts are equivalent to a coarse parallactic or stereo-radargrammetric DEM. Thus, the relation between the signal first-second year monostatic shifts and the second year bistatic shifts is the same as the relation between the geometrical first-second year monostatic shifts and the second year bistatic shifts:

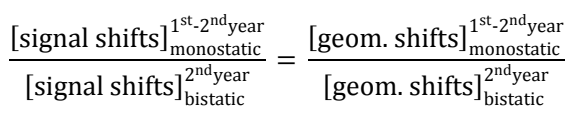

The equivalent precise monostatic signal shifts can thus be derived following:

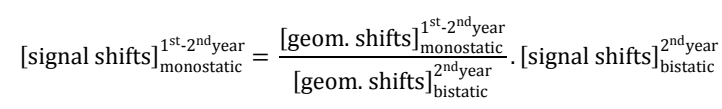

The obtained shifts are then used to resample the slave data to the master geometry. Once both scenes are coregistered, the common part is cropped. The interferogram is then calculated and unwrapped using the Minimum Cost Flow algorithm [6], which was used successfully during the SRTM mission.

\subsection{Phase unwrapping consistency check}

At this point, two unwrapped phases are available. It is not possible to compare them directly since they have different baselines, they have to be transformed into slant range heights using fine height of ambiguity maps. Once in height domain, the consistency check can take place. As a matter of fact, a phase unwrapping error is then detectable since the height difference will vary. A residual trend coming for example from baselines errors, different tropospheric delays, different absolute height offsets is compensated. Then, height differences are calculated and a histogram of those differences is computed (Figure 3). When the phase is unwrapped successfully, in other words error free, both heights have a constant offset so that the histogram should have only one dominant peak and phase unwrapping is consistent. On the contrary, as long as this histogram presents several peaks, phase unwrapping is not consistent 


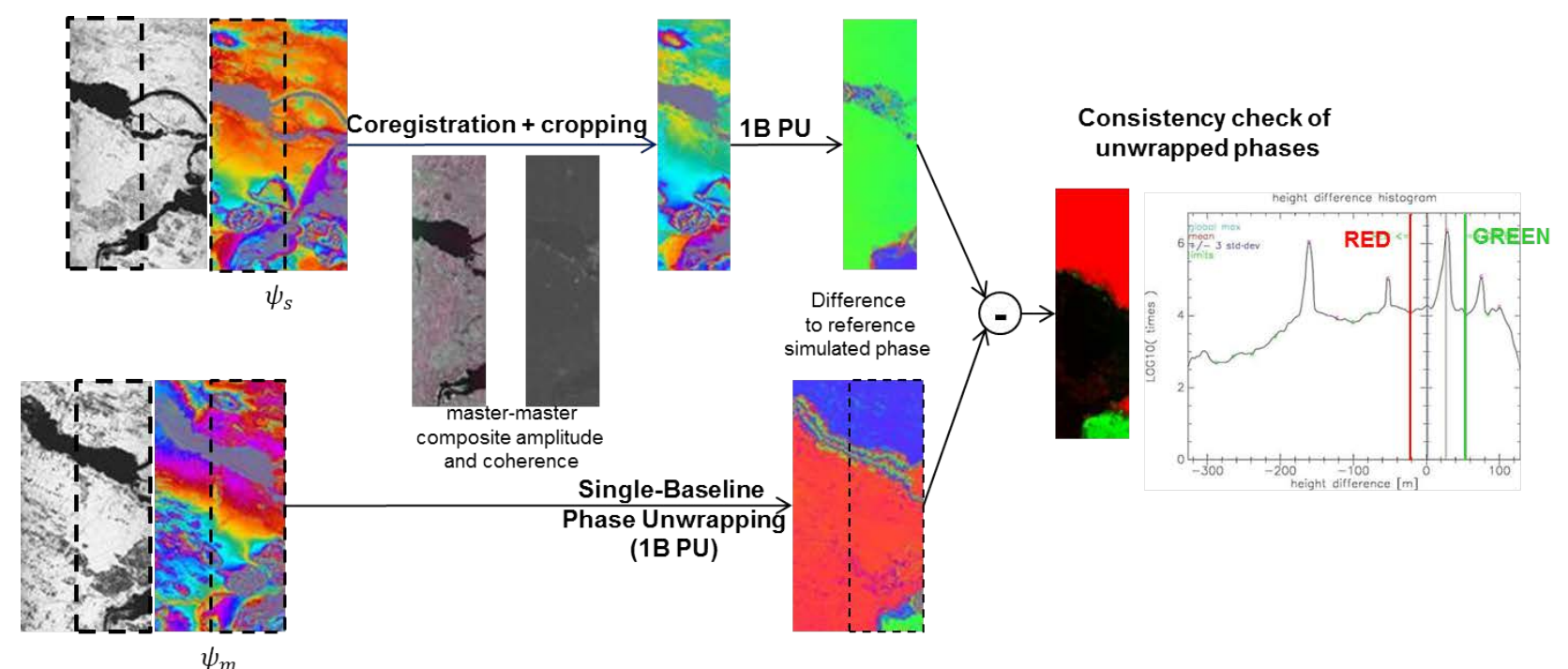

Figure 3: Overview of the dual-baseline interferometric chain:

slave interferogram coregistration, cropping, phase unwrapping and consistency check

and a mask of inconsistent regions is generated. When consistency is proved, no further processing is necessary for this slave since no correction is needed. Otherwise, the unwrapping errors have to be removed.

\subsection{Phase unwrapping errors correction}

The phase unwrapping errors can be detected using both the master and slave unwrapped phases converted to height but only with this information, no further correction is feasible. Indeed, it cannot be clearly determined in which unwrapped phase the error is, it may even be in both unwrapped phases. A third reliable measurement is thus needed and it is obtained forming the differential interferogram.

The differential interferogram means here the product conjugate complex of both master and slave interferograms (eq. 4) in order to obtain a third one with a higher equivalent height of ambiguity $h_{2 \pi}$ given by equation 5 [1].

$$
\begin{gathered}
I_{d}=I_{m} \cdot I_{s}^{*} \\
h_{2 \pi}^{d}=\left(\frac{1}{h_{2 \pi}^{m}}-\frac{1}{h_{2 \pi}^{s}}\right)^{-1}
\end{gathered}
$$

This differential interferogram is thus easier to unwrap but exhibits higher noise. That's why a fringe direction adaptive filtering is performed before its unwrapping. Once it is unwrapped, its correctness is checked using the stereoradargrammetric phase derived from the coregistration shifts and possible phase unwrapping errors are eliminated with these coarse absolute height information.

This correction only changes the ambiguity band and has several levels of complexity. It will not be further explained here. A full description of the algorithms can be found in [3].

\subsection{Mosaicking of the different corrected parts}

The corrected phases coming from the different overlapping slave interferograms are then mosaicked to rebuild the entire scene. Remaining offsets between the different parts are corrected. In the overlapping areas of the corrected phases, the resulting unwrapped phase depends on how every corrected phase has been obtained. There, the corrected values can be either equal (straightforward) or different by a multiple of $2 \pi$ since only the ambiguity band is changed during the correction step. In the latter case, the final corrected phase is chosen according to the correction quality.

\section{RESULTS}

Figure 4 shows different typical problems for the phase unwrapping in the TanDEM-X mission. The first case is a part of the border between Lesotho and South Africa which is a natural wall of more than $500 \mathrm{~m}$ high. The second example is a typical mountainous terrain. The last case is a river: every river (thus completely incoherent) which cuts somehow the scene in two parts may cause phase unwrapping errors. Images (a) and (b) are the comparison with the SRTM phase before (a) and after (b) the dualbaseline interferometric chain: the green color indicates agreement in terms of ambiguity band. Note that the second scene of the first example (natural wall) has been corrected with the help of two scenes (Figure 4.1.e).

Table 1 exhibits the improvement of the phase unwrapping quality ratio. This ratio is the main quality ratio of the ITP to assess the phase unwrapping. It is more or less the percentage of pixels correctly unwrapped w.r.t. the radargrammetric estimate [7]. The dual-baseline interferometric processing clearly improves the phase unwrapping and thus the final quality of the DEM. 
1. natural wall:

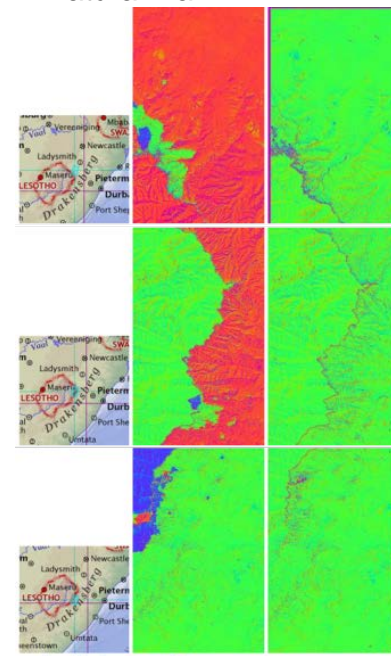

(a)

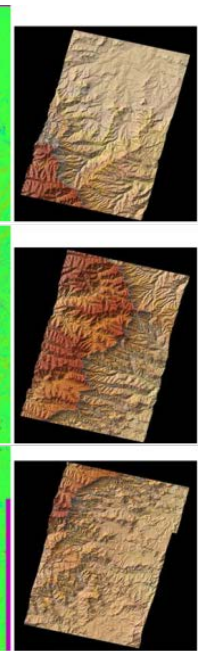

(c)

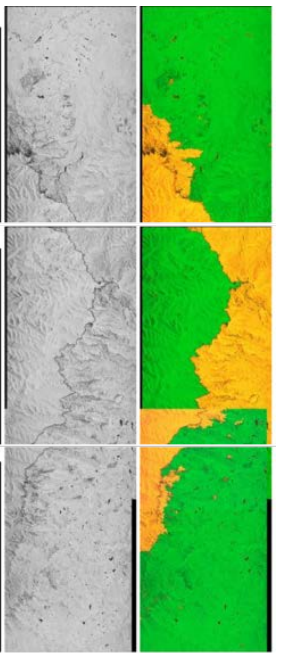

(d)

2. mountainous terrain:

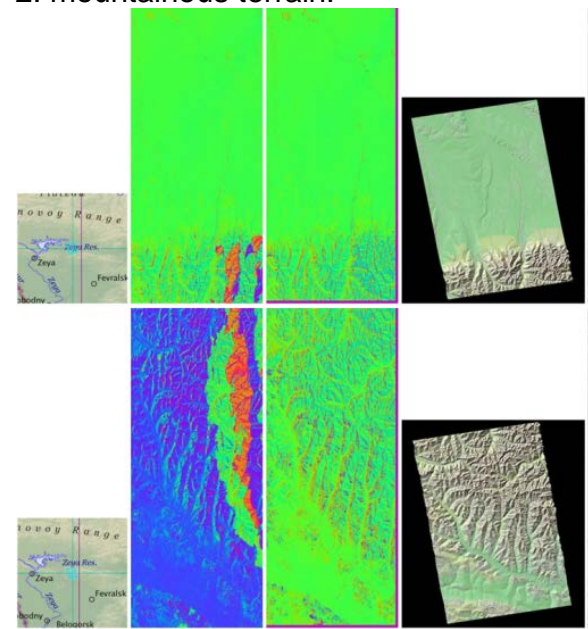

(a) (b)

(c)

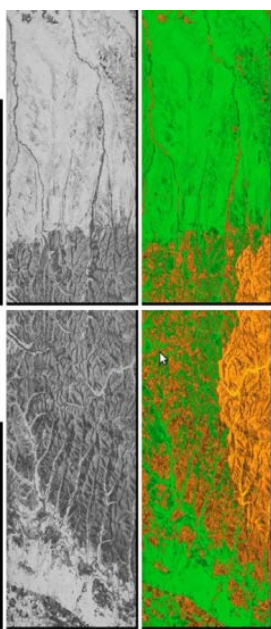

(d)

3. river:

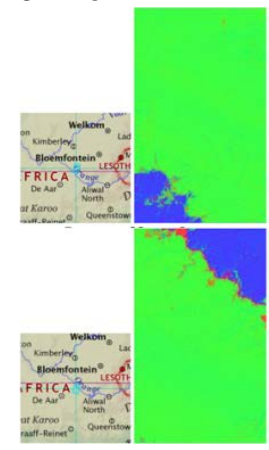

(a)

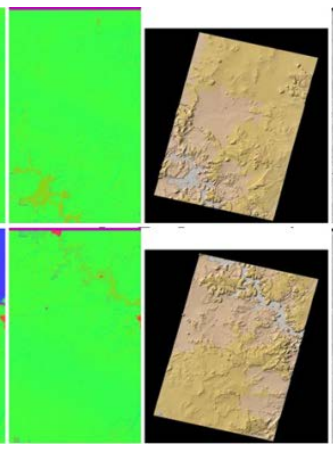

(b) (c)

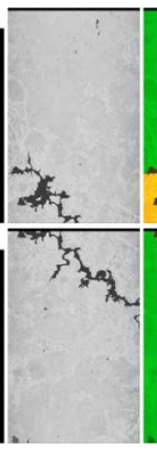

(d)

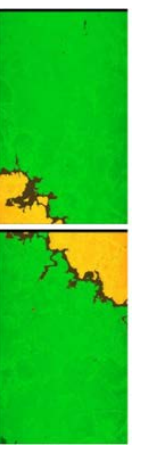

(e)

Figure 4: Results of the dual-baseline processing chain for typical phase unwrapping problems for the TanDEM-X mission: 1 natural wall, 2 mountainous terrain, 3 river; the different images are respectively the differences to SRTM phase before (a) and after (b) the correction, (c) the resulting raw DEM, (d) the coherence of the master and slave interferograms and (e) the detected regions to correct in yellow.

\begin{tabular}{|c|c|c|}
\hline Scenes & PU ratio before & PU ratio after \\
\hline natural wall 1 & $88,3 \%$ & $99,9 \%$ \\
\hline natural wall 2 & $52,7 \%$ & $100 \%$ \\
\hline natural wall 3 & $89,4 \%$ & $100 \%$ \\
\hline mountainous terrain 1 & $96,6 \%$ & $99,8 \%$ \\
\hline mountainous terrain 2 & $72,8 \%$ & $99,9 \%$ \\
\hline river 1 & $92,6 \%$ & $100 \%$ \\
\hline river 2 & $87,0 \%$ & $100 \%$ \\
\hline
\end{tabular}

Table 1: Phase unwrapping quality ratio before and after the dualbaseline interferometric chain

\section{CONCLUSION}

We presented the dual-baseline interferometric processing chain of the ITP for the second year of the TanDEM-X. Different scenes or supporting CoSSCs acquired during the first year are used to improve the critical phase unwrapping step and thus deliver a raw DEM of good quality. Our method works well as long as the terrain is not too extreme (to be treated in the third mission year).

\section{REFERENCES}

[1] G. Krieger, A. Moreira, H. Fiedler, I. Hajnsek, M. Werner, M. Younis, and M. Zink, "TanDEM-X: A satellite formation for High-Resolution SAR Interferometry”, TGARS, vol. 45, No 11. November 2007

[2] N. Yague-Martinez, C. Rossi, M. Lachaise, F. RodriguezGonzalez, T. Fritz, and H. Breit, "Interferometric processing algorithms of TanDEM-X data”, Proc. IGARSS 2010, Honolulu, July 2010

[3] M. Lachaise, T. Fritz, U. Balss, and R. Bamler, "Phase unwrapping correction with dual-baseline data for the TanDEM-X mission”, to be published in Proc. IGARSS 2012, Munich, July 2012

[4] G. Ferraiuolo, V. Pascazio, and G. Schirinzi, "Maximum a posteriori estimation of height profiles in InSAR imaging," GRSL, vol. 1, no. 2, pp. 66-70, April 2004

[5] N. Yague-Martinez, M. Eineder, H. Breit, T. Fritz, and R. Brcic, "TanDEM-X Mission: SAR Image Coregistration Aspects”, Proc. EUSAR 2010, Aachen, 2010

[6] M. Eineder, M. Hubig, and B. Milcke, "Unwrapping large interferograms using the minimum cost flow algorithm”, Proc. IGARSS 1998, Seattle, July 1998

[7] C. Rossi, F. Rodriguez Gonzalez, T. Fritz, N. Yague Martinez, and M. Eineder, "Calibrated TanDEM-X Raw DEM Generation”, ISPRS J. Photogramm. Remote Sens., under review, 2012 